This item was submitted to Loughborough's Research Repository by the author.

Items in Figshare are protected by copyright, with all rights reserved, unless otherwise indicated.

\title{
Adaptive nonlinear equalizer using a mixture of Gaussian-based online density estimator
}

PLEASE CITE THE PUBLISHED VERSION

http://dx.doi.org/10.1109/TVT.2014.2313458

PUBLISHER

(C) IEEE

VERSION

AM (Accepted Manuscript)

LICENCE

CC BY-NC-ND 4.0

\section{REPOSITORY RECORD}

Chen, Hao, Yu Gong, Xia Hong, and Sheng Chen. 2019. "Adaptive Nonlinear Equalizer Using a Mixture of Gaussian-based Online Density Estimator”. figshare. https://hdl.handle.net/2134/25656. 


\title{
Adaptive Equalizer Using a Mixture of
}

\section{Gaussians Based On-Line Density Estimator}

\author{
Hao Chen, Student Member, IEEE, Yu Gong, Member, IEEE, Xia Hong, Senior \\ Member, IEEE, and Sheng Chen, Fellow, IEEE
}

\begin{abstract}
This paper introduces a new adaptive nonlinear equalizer relying on a radial basis function model, which is designed based on the minimum bit error rate (MBER) criterion, in the system setting of the intersymbol interference channel plus a co-channel interference. Our proposed algorithm is referred to as the on-line mixture of Gaussians estimator aided MBER (OMG-MBER) equalizer. Specifically, a mixture of Gaussians based probability density function (PDF) estimator is used to model the PDF of the decision variable, for which a novel on-line PDF update algorithm is derived to track the incoming data. With the aid of this novel on-line mixture of Gaussians based sample-by-sample updated PDF estimator, our adaptive nonlinear equalizer is capable of updating its equalizer's parameters sample by sample to aim directly at minimizing the equalizer's achievable bit error rate (BER). The proposed OMG-MBER equalizer significantly outperforms the existing on-line nonlinear MBER equalizer, known as the least bit error rate equalizer, in terms of both the convergence speed and the achievable BER, as is confirmed in our simulation study.
\end{abstract}

\section{Index Terms}

Adaptive nonlinear equalizer, radial basis function, minimum bit error rate, probability density function, mixture of Gaussians

Hao Chen and Xia Hong are with the School of Systems Engineering, University of Reading, Reading RG6 6AY, UK (E-mails: hao.chen@pgr.reading.ac.uk, x.hong@reading.ac.uk).

Yu Gong is with the School of Electronic, Electrical and Systems Engineering, Loughborough University, Loughborough LE11 3TU, UK (E-mail: alex.yugong@gmail.com).

Sheng Chen is with Electronics and Computer Science, University of Southampton, Southampton SO17 1BJ, UK (Email: sqc@ecs.soton.ac.uk), and also with King Abdulaziz University, Jeddah 21589, Saudi Arabia.

This research is sponsored by the UK Engineering and Physical Sciences Research Council and DSTL under the grant number EP/H012516/1. 


\section{INTRODUCTION}

Channel equalization as a standard approach in communications to combat the dispersive effects of a channel has been very well studied [1]-[4]. Both linear and nonlinear equalizers have been proposed [5]-[9], and the majority of them are based on the minimum mean-squareerror (MMSE) criterion. The MMSE equalizer has the advantage of easy implementation with good performance. The MMSE linear equalizer also has a nature link to adaptive filters [10], and admits a very simple yet powerful on-line adaptation by the least mean square (LMS) algorithm [11]. However, the MMSE criterion is not equivalent to the minimum bit error rate (MBER) criterion, and the latter is the ultimate performance criterion for communication channel equalization [12]-[32]. Equalizers based on the MBER criterion are thus of great interests and have attracted considerable attentions [14], [20]-[23], [33]-[35]. The least bit error rate (LBER) nonlinear equalizer for binary transmission was proposed in [33], which was shown to achieve significant performance gain over both the adaptive linear and nonlinear MMSE equalizers.

The equalization problem can alternatively be regarded as a classification problem, where the task of the equalizer is to determine the decision boundaries for separating the different classes of data. It is shown in [33] that the adaptive MBER nonlinear equalizer achieves the decision boundary that is close to the optimum Bayesian equalizer, and so is its equalization performance. While both the batch and sample-by-sample adaptive MBER equalizers were proposed in [33], the latter, referred to as the LBER, is of particular interest since it is an on-line adaptive algorithm with very low computational complexity and is capable of tracking the channel variation well. In order to update the gradient of the bit error rate (BER) with the new coming data, which is required for adapting the MBER equalizer's parameters, it is necessary to adaptively estimate the probability density function (PDF) of the signed decision variable constructed based on the desired signal and the equalizer output. The LBER nonlinear equalizer [33] adopts a one-sample or single Gaussian kernel estimate for the PDF of the signed decision variable to realize sampleby-sample adaptation of the equalizer's parameters, in a manner similar to the LMS algorithm which uses a one sample estimate for the mean square error [10].

The performance of an adaptive MBER equalizer is to a large extent determined by the on-line PDF estimator employed. Despite of its computational simplicity and its superior performance over the adaptive MMSE equalizer, the LBER equalizer of [33] has a drawback, owing to the fact that it adopts a one-sample PDF estimate which is stochastic by nature and is very sensitive to the noise in the received signal sample. In statistical data learning, there exist large amount of works 
[36]-[41] using the Gaussian mixture model to estimate PDF. These kernel density estimators based on a mixture of Gaussians however are batch learning algorithms by nature and they are unsuitable for on-line applications. In this paper, we also use a kernel density estimator, consisting of a small number of Gaussian kernels to estimate the PDF of the signed decision variable. Our novel contribution is to propose a new on-line mixture of Gaussians estimator, referred to as the OMG, to update the PDF estimate sample by sample. To be specific, a new Gaussian kernel is formed for every new data and it is then merged with the "nearest" existing Gaussian kernel in the kernel density estimator. With the aid of this OMG for on-line estimation of the signed decision variable's PDF, the nonlinear equalizer's parameters can be adapted sample by sample in the way similar to the LBER nonlinear equalizer of [33]. We refer to this new adaptive MBER nonlinear equalizer as the OMG aided MBER (OMG-MBER) algorithm. Because the proposed OMG-MBER equalizer relies on a much more accurate on-line PDF estimate, unlike the onesample PDF estimator of the LBER algorithm, it outperforms the LBER nonlinear equalizer in terms of both the convergence speed and the equalizer's achievable BER. A simulation study is carried out and the results obtained confirm that the OMG-MBER equalizer significantly improve the equalization performance, compared with the existing LBER equalizer.

This paper is organized as follows. Section II introduces the equalisation problem in the setting of the intersymbol interference channel plus a co-channel interference (CCI) as well as reviews the existing adaptive MMSE and MBER equalizers. Section III details our proposed novel OMG-MBER nonlinear equalizer. The simulation results are presented in Section IV, and our conclusions are given in Section V.

\section{PRELIMINARIES}

We first introduce the channel model and then review the adaptive equalizers based on the MMSE and MBER criteria.

\section{A. System Model}

We assume without loss of generality that the system suffers from one CCI. The received signal sample at symbol index $k$ is given by [42]

$$
\begin{aligned}
r(k) & =\bar{r}(k)+n(k)=\bar{r}_{0}(k)+\bar{r}_{1}(k)+n(k) \\
& =\sum_{i=0}^{n_{0}-1} a_{0, i} b_{0}(k-i)+\sum_{i=0}^{n_{1}-1} a_{1, i} b_{1}(k-i)+n(k),
\end{aligned}
$$


where $n(k)$ is the additive white Gaussian noise (AWGN) with zero mean and variance $E\left[n^{2}(k)\right]=$ $\sigma_{n}^{2}, \bar{r}(k), \bar{r}_{0}(k)$ and $\bar{r}_{1}(k)$ are the noise-free received signal, desired signal and interfering signal, respectively, while $a_{0, i}, 0 \leq i \leq n_{0}-1$, and $a_{1, i}, 0 \leq i \leq n_{1}-1$, are the coefficients for the channel and co-channel, respectively, $b_{0}(k)$ and $b_{1}(k)$ are the uncorrelated binary desired and interfering data, respectively, taking value from the set $\{ \pm 1\}$.

We assume that the equalizer length is $m$, namely, the equalization is based on the received vector of the $m$ most recently received signal samples given by

$$
\mathbf{r}(k)=[r(k) r(k-1) \cdots r(k-m+1)]^{\mathrm{T}} .
$$

Then (1) can be expressed in the matrix form as

$$
\mathbf{r}(k)=\overline{\mathbf{r}}(k)+\mathbf{n}(k)=\overline{\mathbf{r}}_{0}(k)+\overline{\mathbf{r}}_{1}(k)+\mathbf{n}(k)=\mathbf{H}_{0} \mathbf{b}_{0}(k)+\mathbf{H}_{1} \mathbf{b}_{1}(k)+\mathbf{n}(k),
$$

where $\mathbf{n}(k)=[n(k) n(k-1) \cdots n(k-m+1)]^{\mathrm{T}}$ is the AWGN vector, $\mathbf{b}_{0}(k)=\left[b_{0}(k) b_{0}(k-\right.$ 1) $\left.\cdots b_{0}\left(k-n_{0}-m+2\right)\right]^{\mathrm{T}}$ and $\mathbf{b}_{1}(k)=\left[b_{1}(k) b_{1}(k-1) \cdots b_{1}\left(k-n_{1}-m+2\right)\right]^{\mathrm{T}}$ are the desired and interfering signal vectors, respectively, while $\mathbf{H}_{0} \in \mathbb{R}^{m \times\left(m+n_{0}-1\right)}$ and $\mathbf{H}_{1} \in \mathbb{R}^{m \times\left(m+n_{1}-1\right)}$ are the desired and interfering channel matrices, respectively, given by

$$
\mathbf{H}_{i}=\left[\begin{array}{ccccccc}
a_{i, 0} & a_{i, 1} & \cdots & a_{i, n_{i}-1} & 0 & \cdots & 0 \\
0 & a_{i, 0} & a_{i, 1} & \cdots & a_{i, n_{i}-1} & \ddots & \vdots \\
\vdots & \ddots & \ddots & \ddots & \cdots & \ddots & 0 \\
0 & \cdots & 0 & a_{i, 0} & a_{i, 1} & \cdots & a_{i, n_{i}-1}
\end{array}\right],
$$

for $i=0$ and 1 . The equalization output can be expressed as

$$
\widehat{b}_{0}(k-d)=\operatorname{sgn}(y(k)) \text { with } y(k)=f(\mathbf{r}(k) ; \mathbf{w}),
$$

where $f(. ;$.$) denotes the equalizer function, w contains all the equalizer's adjustable parameters,$ and $d$ is the decision delay.

As $b_{0}(k)$ and $b_{1}(k)$ are binary signals, there are $N_{0}=2^{m+n_{0}-1}$ and $N_{1}=2^{m+n_{1}-1}$ possible combinations or "states" for $\mathbf{b}_{0}(k)$ and $\mathbf{b}_{1}(k)$, respectively. The $j$-th state of $\mathbf{b}_{0}(k)$ is denoted as $\mathbf{b}_{0, j}$, where $1 \leq j \leq N_{0}$, while the $l$-th state of $\mathbf{b}_{1}(k)$ is denoted as $\mathbf{b}_{1, l}$, where $1 \leq l \leq N_{1}$. Accordingly, there are $N_{0}$ and $N_{1}$ states for $\overline{\mathbf{r}}_{0}(k)$ and $\overline{\mathbf{r}}_{1}(k)$, denoted as $\left\{\overline{\mathbf{r}}_{0, j}=\mathbf{H}_{0} \mathbf{b}_{0, j}\right\}_{j=1}^{N_{0}}$ and $\left\{\overline{\mathbf{r}}_{1, l}=\mathbf{H}_{1} \mathbf{b}_{1, l}\right\}_{l=1}^{N_{1}}$, respectively. Consequently, there are $N_{r}=N_{0} \times N_{1}$ states for $\overline{\mathbf{r}}(k)$, which we denote as $\mathbf{R}=\left\{\overline{\mathbf{r}}_{i}\right\}_{i=1}^{N_{r}}$. $\mathbf{R}$ can be divided into two sub-sets, $\mathbf{R}_{+}$and $\mathbf{R}_{-}$, corresponding to the $d$-th element of $\mathbf{b}_{0, j}$, denoted as $b_{0, j}^{(d)}$, being +1 and -1 , respectively. Thus $b_{0, j}^{(d)}$ serves as the 
"class" label for $\mathbf{r}(k) \in \mathbb{R}^{m}$, and the equalization (5) is equivalent to a classification process, which partitions the $m$-dimensional observation space by the decision boundary defined as

$$
f(\mathbf{r} ; \mathbf{w})=0 .
$$

The optimal Bayesian equalizer $y_{B}(k)=f_{B}(\mathbf{r}(k) ; \mathbf{w})$, given the channel and co-channel, is expressed by [42], [43]:

$$
\begin{aligned}
f_{B}(\mathbf{r}(k) ; \mathbf{w}) & =\sum_{j=1}^{N_{0}} \sum_{l=1}^{N_{1}} \frac{b_{0, j}^{(d)}}{\left(2 \pi \sigma_{n}^{2}\right)^{m / 2} N_{0} N_{1}} e^{-\frac{\left\|\mathbf{r}(k)-\overline{\mathbf{r}}_{0, j}-\overline{\mathbf{r}}_{1, l}\right\|^{2}}{2 \sigma_{n}^{2}}} \\
& =\sum_{i=1}^{N_{r}} \frac{b_{i}^{(d)}}{\left(2 \pi \sigma_{n}^{2}\right)^{m / 2} N_{r}} e^{-\frac{\left\|\mathbf{r}(k)-\overline{\mathbf{r}}_{i}\right\|^{2}}{2 \sigma_{n}^{2}}}
\end{aligned}
$$

where $b_{i}^{(d)}$ is the class label for $\overline{\mathbf{r}}_{i}$. However, the computational complexity of this Bayesian solution is often too high, because the size of $\mathbf{R}$ is typically huge.

\section{B. Equalizer Based on MMSE Criterion}

Due to its mathematical tractability, the most widely adopted criterion for training an equalizer is the MMSE criterion, and the well-known LMS algorithm [11] offers an adaptive algorithm for training a linear equalizer based on the MMSE criterion. The LMS algorithm can also be extended to train a nonlinear equalizer according to

$$
\mathbf{w}(k)=\mathbf{w}(k-1)+\eta\left(b_{0}(k-d)-y(k)\right) \frac{\partial f(\mathbf{r}(k) ; \mathbf{w}(k-1))}{\partial \mathbf{w}},
$$

where $\eta$ is a small positive step size.

The adaptive non-linear equalizer design based on the radial basis function (RBF) network was well studied [43], and the RBF equalizer output is given by

$$
y(k)=f(\mathbf{r}(k) ; \mathbf{w})=\sum_{j=1}^{n_{c}} \alpha_{j} e^{-\frac{\left\|\mathbf{r}(k)-\mathbf{c}_{j}\right\|^{2}}{\sigma_{j}^{2}}},
$$

where $n_{c}$ is the number of RBF nodes employed, and the equalization parameter vector $\mathbf{w}$ consists of all the RBF weights $\alpha_{j}$, variances $\sigma_{j}^{2}$ and centers $\mathbf{c}_{j}$. The dimension of $\mathbf{w}$ is therefore $n_{c} \times(m+2)$. The partial derivatives of the equalizer output with respect to the equalizer's 
parameters $\mathbf{w}$ in (8) are given for $1 \leq j \leq n_{c}$ by

$$
\left\{\begin{array}{l}
\frac{\partial f(\mathbf{r}(k) ; \mathbf{w})}{\partial \alpha_{j}}=e^{-\frac{\left\|\mathbf{r}(k)-\mathbf{c}_{j}\right\|^{2}}{\sigma_{j}^{2}}} \\
\frac{\partial f(\mathbf{r}(k) ; \mathbf{w})}{\partial \sigma_{j}^{2}}=\alpha_{j} e^{-\frac{\left\|\mathbf{r}(k)-\mathbf{c}_{j}\right\|^{2}}{\sigma_{j}^{2}}} \frac{\left\|\mathbf{r}(k)-\mathbf{c}_{j}\right\|^{2}}{\sigma_{j}^{4}} \\
\frac{\partial f(\mathbf{r}(k) ; \mathbf{w})}{\partial \mathbf{c}_{j}}=2 \alpha_{j} e^{-\frac{\left\|\mathbf{r}(k)-\mathbf{c}_{j}\right\|^{2}}{\sigma_{j}^{2}}} \frac{\mathbf{r}(k)-\mathbf{c}_{j}}{\sigma_{j}^{2}}
\end{array}\right.
$$

For the linear equalizer

$$
y(k)=f_{L}(\mathbf{r}(k) ; \mathbf{w})=\mathbf{w}^{\mathrm{T}} \mathbf{r}(k)
$$

(8) returns to its original LMS form

$$
\mathbf{w}(k)=\mathbf{w}(k-1)+\eta\left(b_{0}(k-d)-y(k)\right) \mathbf{r}(k) .
$$

\section{Equalizer Based on MBER Criterion}

However, the ultimate goal of equalization is to minimize the BER [12]-[16]. Many studies [20]-[23], [33]-[35] have demonstrated that the MBER equalizer can significantly improve the performance compared with the traditional equalizer design based on the MMSE criterion. Specifically, for the linear equalizer (11), the MBER design has been extensively studied [13][16], [18], [20], and adaptive MBER linear equalizer has been proposed based on the LBER algorithm [21], [23]. Moreover, the MBER design and its adaptive LBER algorithm have been extended to the generic nonlinear equalizer of (5) [33], [34].

The error probability of the equalizer (5) can be obtained as [33]

$$
P_{E}(\mathbf{w})=\operatorname{Prob}\left\{\operatorname{sgn}\left(b_{0}(k-d)\right) y(k)<0\right\} .
$$

Defining the signed decision variable as $y_{s}(k)=\operatorname{sgn}\left(b_{0}(k-d)\right) y(k)$, we have

$$
P_{E}(\mathbf{w})=\int_{-\infty}^{0} p_{y}\left(y_{s}\right) d y_{s}
$$

where $p_{y}\left(y_{s}\right)$ is the PDF of $y_{s}(k)$. Linearizing the equalizer around $\overline{\mathbf{r}}(k)$ yields [33]

$$
y(k)=f(\overline{\mathbf{r}}(k)+\mathbf{n}(k) ; \mathbf{w}) \approx f(\overline{\mathbf{r}}(k) ; \mathbf{w})+e(k)=\bar{y}(k)+e(k),
$$


where $e(k)$ is an equivalent zero-mean Gaussian noise with variance $\rho^{2}$. Noting that the noise-free received signal $\bar{y}(k)$ takes value from the finite set, namely, $\bar{y}(k) \in Y_{f}=\left\{\bar{y}_{i}=f\left(\overline{\mathbf{r}}_{i} ; \mathbf{w}\right)\right\}_{i=1}^{N_{r}}$, the PDF of $y_{s}(k)$ can be approximated as [33]

$$
p_{y}\left(y_{s}\right) \approx \frac{1}{N_{r} \sqrt{2 \pi} \rho} \sum_{i=1}^{N_{r}} e^{-\frac{\left(y_{s}-\operatorname{sgn}\left(b_{i}^{(d)}\right) \bar{y}_{i}\right)^{2}}{2 \rho^{2}}} .
$$

Substituting (16) into (14) then yields the following error probability of the equalizer

$$
P_{E}(\mathbf{w}) \approx \frac{1}{N_{r} \sqrt{2 \pi}} \sum_{i=1}^{N_{r}} \int_{\bar{g}_{i}(\mathbf{w})}^{\infty} e^{-\frac{x_{i}^{2}}{2}} d x_{i}=\frac{1}{N_{r}} \sum_{i=1}^{N_{r}} Q\left(\bar{g}_{i}(\mathbf{w})\right)
$$

where

$$
\begin{aligned}
Q(x) & =\frac{1}{\sqrt{2 \pi}} \int_{x}^{\infty} e^{-\frac{y^{2}}{2}} d y, \\
\bar{g}_{i}(\mathbf{w}) & =\frac{\operatorname{sgn}\left(b_{i}^{(d)}\right) f\left(\overline{\mathbf{r}}_{i} ; \mathbf{w}\right)}{\rho} .
\end{aligned}
$$

Then, the gradient of $P_{E}(\mathbf{w})$ can be approximated as

$$
\nabla P_{E}(\mathbf{w}) \approx-\frac{1}{N_{r} \sqrt{2 \pi}} \sum_{i=1}^{N_{r}} e^{-\frac{\bar{y}_{i}^{2}}{2 \rho^{2}}} \frac{\partial \bar{g}_{i}(\mathbf{w})}{\partial \mathbf{w}}=-\frac{1}{N_{r} \sqrt{2 \pi}} \sum_{i=1}^{N_{r}} e^{-\frac{\bar{y}_{i}^{2}}{2 \rho^{2}} \operatorname{sgn}\left(b_{i}^{(d)}\right) \frac{\partial f\left(\overline{\mathbf{r}}_{i} ; \mathbf{w}\right)}{\partial \mathbf{w}} .}
$$

Thus, if the channel and co-channel are known, the equalizer parameters can be updated by minimizing the approximate BER in (17), leading to the MBER updating rule

$$
\mathbf{w}(l)=\mathbf{w}(l-1)-\eta \nabla P_{E}(\mathbf{w}(l-1)) .
$$

In practice, the channel and co-channel are unknown. Thus, the set $\mathbf{R}$ and hence the set $Y_{f}$ are unknown. The key to developing an effective adaptive algorithm for implementing the MBER design is to estimate the PDF $p_{y}\left(y_{s}\right)$ of the signed decision variable $y_{s}(k)$ based on the training data $D_{K}=\left\{\mathbf{r}(k), b_{0}(k-d)\right\}_{k=1}^{K}$ [33]. Specifically, given $D_{K}$, the Parzen window or kernel density estimate of the PDF $p_{y}\left(y_{s}\right)$ is given as [33]

$$
\widehat{p}_{y}\left(y_{s}\right)=\frac{1}{K \sqrt{2 \pi} \rho} \sum_{k=1}^{K} e^{-\frac{\left(y_{s}-\operatorname{sgn}\left(b_{0}(k-d)\right) y(k)\right)^{2}}{2 \rho^{2}}} .
$$

Substituting (22) into (14) yields the estimated error probability $\widehat{P}_{E}(\mathbf{w})$, the gradient of which is given by

$$
\nabla \widehat{P}_{E}(\mathbf{w})=-\frac{1}{K \sqrt{2 \pi} \rho} \sum_{k=1}^{K} e^{-\frac{y^{2}(k)}{2 \rho^{2}}} \operatorname{sgn}\left(b_{0}(k-d)\right) \frac{\partial f(\mathbf{r}(k) ; \mathbf{w})}{\partial \mathbf{w}} .
$$


This leads to the block adaptive MBER algorithm [33]

$$
\begin{aligned}
\mathbf{w}(l) & =\mathbf{w}(l-1)-\eta \nabla \widehat{P}_{E}(\mathbf{w}(l-1)) \\
& =\mathbf{w}(l-1)+\frac{\eta}{K \sqrt{2 \pi} \rho} \sum_{k=1}^{K} e^{-\frac{y^{2}(k)}{2 \rho^{2}}} \operatorname{sgn}\left(b_{0}(k-d)\right) \frac{\partial f(\mathbf{r}(k) ; \mathbf{w}(l-1))}{\partial \mathbf{w}} .
\end{aligned}
$$

To realize a sample by sample adaptation, the nonlinear LBER algorithm [33] only uses a single data point to estimate the PDF

$$
\widetilde{p}_{y}\left(y_{s}, k\right)=\frac{1}{\sqrt{2 \pi} \rho} e^{-\frac{\left(y_{s}-\operatorname{sgn}\left(b_{0}(k-d)\right) y(k)\right)^{2}}{2 \rho^{2}}} .
$$

With this "instantaneous" PDF estimate, we have a "one-sample" error probability estimate $\widetilde{P}_{E}(\mathbf{w}, k)$ with the stochastic gradient given by

$$
\nabla \widetilde{P}_{E}(\mathbf{w}, k)=-\frac{1}{\sqrt{2 \pi} \rho} e^{-\frac{y^{2}(k)}{2 \rho^{2}} \operatorname{sgn}\left(b_{0}(k-d)\right) \frac{\partial f(\mathbf{r}(k) ; \mathbf{w})}{\partial \mathbf{w}} .}
$$

This leads to the stochastic gradient adaptive algorithm known as the LBER algorithm [33]

$$
\mathbf{w}(k)=\mathbf{w}(k-1)+\frac{\eta}{\sqrt{2 \pi} \rho} e^{-\frac{y^{2}(k)}{2 \rho^{2}}} \operatorname{sgn}\left(b_{0}(k-d)\right) \frac{\partial f(\mathbf{r}(k) ; \mathbf{w}(k-1))}{\partial \mathbf{w}},
$$

in which the equalizer parameter vector is adjusted sample by sample, in a similar manner to the LMS algorithm in (12).

\section{On-Line MiXture of Gaussians Estimator Aided MBER Equalizer}

In order to realize a sample-by-sample adaptive MBER equalizer, the LBER algorithm [33] uses a single sample to estimate the PDF on-line. Our OMG-MBER adopts a novel mixture of Gaussians based on-line PDF estimator. Specifically, we derive the kernel PDF estimator based on a mixture of Gaussians with a small number of mixtures, which is capable of adapting the PDF estimate sample by sample. With the aid of this on-line kernel density estimation algorithm, our adaptive MBER equalizer is capable of updating its equalizer's parameters sample by sample, similar to the LBER algorithm. Since our OMG-MBER algorithm relies on a much more accurate one-line PDF estimate, unlike the one-sample PDF of the LBER algorithm, we expect our OMGMBER will outperform the LBER, at the expense of negligibly small increase in complexity. 


\section{A. On-Line Kernel Based PDF Estimator}

Our goal is to derive an on-line PDF estimator that updates the estimate of $p_{y}\left(y_{s}\right)$ as each new data $y_{s}(k)$ is received. We consider the PDF estimator based on the mixture of $M$ Gaussians [37] given by

$$
\begin{gathered}
\widehat{p}_{y}\left(y_{s}\right)=\widehat{p}^{(M)}\left(y_{s} ; \boldsymbol{\lambda}_{M}, \boldsymbol{\mu}_{M}, \boldsymbol{\rho}_{M}\right)=\sum_{j=1}^{M} \lambda_{j} G\left(y_{s} ; \mu_{j}, \rho_{j}\right), \\
\text { s.t. } \lambda_{j}>0,1 \leq j \leq M \text {, and } \boldsymbol{\lambda}_{M}^{\mathrm{T}} \mathbf{1}_{M}=1,
\end{gathered}
$$

where $\lambda_{j}, \mu_{j}$ and $\rho_{j}$ are the weight, mean and standard deviation of the $j$-th Gaussian kernel, respectively, $\boldsymbol{\lambda}_{M}=\left[\begin{array}{llll}\lambda_{1} & \lambda_{2} \cdots \lambda_{M}\end{array}\right]^{\mathrm{T}}, \boldsymbol{\mu}_{M}=\left[\begin{array}{lll}\mu_{1} & \mu_{2} \cdots \mu_{M}\end{array}\right]^{\mathrm{T}}, \boldsymbol{\rho}_{M}=\left[\begin{array}{lll}\rho_{1} & \rho_{2} \cdots \rho_{M}\end{array}\right]^{\mathrm{T}}, \mathbf{1}_{M}$ is the $M$-dimensional vector whose elements are all equal to one, and

$$
G\left(y_{s} ; \mu_{j}, \rho_{j}\right)=\frac{1}{\sqrt{2 \pi} \rho_{j}} e^{-\frac{\left(y_{s}-\mu_{j}\right)^{2}}{2 \rho_{j}^{2}}} .
$$

At time $k=0$, we have the initial estimate for $p_{y}\left(y_{s}\right)$ given as

$$
\widehat{p}^{(M)}\left(y_{s} ; \boldsymbol{\lambda}_{M}(0), \boldsymbol{\mu}_{M}(0), \boldsymbol{\rho}_{M}(0)\right)=\sum_{j=1}^{M} \frac{1}{M} G\left(y_{s} ; \mu_{j}(0), \rho_{0}\right),
$$

where $\lambda_{j}(0)=\frac{1}{M}$ and $\rho_{j}(0)=\rho_{0}$ for $1 \leq j \leq M$, with $\rho_{0}$ being a predetermined kernel width, while $\mu_{j}(0)$ for $1 \leq j \leq M$ are some randomly drawn positive values. The initialization of the OMG-MBER based nonlinear equalizer will be discussed in more detail later in Subsection III-C.

At time step $k$, the new data point $y_{s}(k)$ is received, and the triplets $\left\{\boldsymbol{\lambda}_{M}, \boldsymbol{\mu}_{M}, \boldsymbol{\rho}_{M}\right\}$ in the PDF estimate

$$
\widehat{p}^{(M)}\left(y_{s} ; \boldsymbol{\lambda}_{M}(k-1), \boldsymbol{\mu}_{M}(k-1), \boldsymbol{\rho}_{M}(k-1)\right)=\sum_{j=1}^{M} \lambda_{j}(k-1) G\left(y_{s} ; \mu_{j}(k-1), \rho_{j}(k-1)\right)
$$

need to be updated accordingly, while keeping the same number of mixtures $M$. A nature way is to place a Gaussian kernel on $y_{s}(k)$ and to merge this new kernel with its nearest existing mixture component $G\left(y_{s} ; \mu_{j^{*}}(k-1), \rho_{j^{*}}(k-1)\right)$, where

$$
j^{*}=\arg \min _{1 \leq j \leq M}\left|y_{s}(k)-\mu_{j}(k-1)\right| .
$$

This can be realized in the following two steps. 
1) Create a temporary $(M+1)$-order model by adding the newly created $(M+1)$-th Gaussian kernel based on $y_{s}(k)$ to the estimate (32) according to

$$
\begin{aligned}
& \widehat{p}^{(M+1)}\left(y_{s} ; \boldsymbol{\lambda}_{M+1}(k), \boldsymbol{\mu}_{M+1}(k), \boldsymbol{\rho}_{M+1}(k)\right)= \\
& \frac{M}{M+1} \sum_{j=1}^{M} \lambda_{j}(k-1) G\left(y_{s} ; \mu_{j}(k-1), \rho_{j}(k-1)\right)+\frac{1}{M+1} G\left(y_{s} ; y_{s}(k), \rho_{0}\right) .
\end{aligned}
$$

Clearly, we set $\lambda_{M+1}(k)=\frac{1}{M+1}$, and for $1 \leq j \leq M$

$$
\lambda_{j}(k)=\frac{M \lambda_{j}(k-1)}{M+1},
$$

to satisfy the constraints $\lambda_{j}(k)>0$ for $1 \leq j \leq M+1$ and $\boldsymbol{\lambda}_{M+1}^{\mathrm{T}}(k) \mathbf{1}_{M+1}=1$, while using $\mu_{M+1}(k)=y_{s}(k), \rho_{M+1}(k)=\rho_{0}$, and for $1 \leq j \leq M$

$$
\begin{gathered}
\mu_{j}(k)=\mu_{j}(k-1), \\
\rho_{j}(k)=\rho_{j}(k-1) .
\end{gathered}
$$

2) Merge the $j^{*}$-th, where $j^{*}$ is determined in (33), and $(M+1)$-th mixtures in the temporary estimate (34) into the single new $j^{*}$-th mixture, so that

$$
\begin{aligned}
\lambda_{j^{*}}(k) G\left(y_{s} ; \mu_{j^{*}}(k), \rho_{j^{*}}(k)\right) \approx & \frac{M \lambda_{j^{*}}(k-1)}{M+1} G\left(y_{s} ; \mu_{j^{*}}(k-1), \rho_{j^{*}}(k-1)\right) \\
& +\frac{1}{M+1} G\left(y_{s} ; y_{s}(k), \rho_{0}\right) .
\end{aligned}
$$

Thus, the new $j^{*}$-th weight $\lambda_{j^{*}}(k)$ is given by

$$
\lambda_{j^{*}}(k)=\frac{M \lambda_{j^{*}}(k-1)}{M+1}+\frac{1}{M+1},
$$

while the new $j^{*}$-th mean and standard deviation, $\mu_{j^{*}}(k)$ and $\rho_{j^{*}}(k)$, are updated by matching the mean and standard deviation of the two mixtures with the new single Gaussian. As is shown in Appendix A, we have the following adaptation rules

$$
\begin{gathered}
\mu_{j^{*}}(k)=\frac{M \lambda_{j^{*}}(k-1)}{M \lambda_{j^{*}}(k-1)+1} \mu_{j^{*}}(k-1)+\frac{1}{M \lambda_{j^{*}}(k-1)+1} y_{s}(k), \\
\rho_{j^{*}}^{2}(k)=\frac{M \lambda_{j^{*}}(k-1)\left(\rho_{j^{*}}^{2}(k-1)+\mu_{j^{*}}^{2}(k-1)\right)}{M \lambda_{j^{*}}(k-1)+1}+\frac{\rho_{0}^{2}+y_{s}^{2}(k)}{M \lambda_{j^{*}}(k-1)+1}-\mu_{j^{*}}^{2}(k) .
\end{gathered}
$$

The PDF of the signed decision variable at sample time $k$ can thus be approximated by

$$
\widehat{p}_{y}\left(y_{s}, k\right)=\widehat{p}^{(M)}\left(y_{s} ; \boldsymbol{\lambda}_{M}(k), \boldsymbol{\mu}_{M}(k), \boldsymbol{\rho}_{M}(k)\right)=\sum_{j=1}^{M} \lambda_{j}(k) G\left(y_{s} ; \mu_{j}(k), \rho_{j}(k)\right),
$$


in which the $j^{*}$-th weight, mean and standard deviation are given in (39), (40) and (41), respectively, while the $j$-th weights, means and standard deviations, where $1 \leq j \leq M$ and $j \neq j^{*}$, are given in (35), (36) and (37), respectively. Note that only $\mu_{j^{*}}(k)$ and $\rho_{j^{*}}^{2}(k)$ contain the new information provided by $y_{s}(k)$. This on-line estimator is a much more accurate estimate of the true PDF $p_{y}\left(y_{s}\right)$ than the one-sample estimate of (25), at the cost of a modest increase in computational requirements, as the number of mixtures $M$ is a very small number.

\section{B. Adaptive MBER Equalizer}

Based on the estimated PDF of the signed decision variable at sample $k$, as is given in (42), the error probability of the equalizer can be expressed as

$$
\begin{aligned}
\widehat{P}_{E}(\mathbf{w}, k) & =\int_{-\infty}^{0} \widehat{p}_{y}\left(y_{s}, k\right) d y_{s} \\
& =\int_{-\infty}^{0}\left(\sum_{j=1, j \neq j^{*}}^{M} \lambda_{j}(k) G\left(y_{s} ; \mu_{j}(k), \rho_{j}(k)\right)+\lambda_{j^{*}}(k) G\left(y_{s} ; \mu_{j^{*}}(k), \rho_{j^{*}}(k)\right)\right) d y_{s} \\
& =\widehat{R}_{E}(\mathbf{w}, k)+\sum_{j=1, j \neq j^{*}}^{M} \lambda_{j}(k) \int_{-\infty}^{0} G\left(y_{s} ; \mu_{j}(k), \rho_{j}(k)\right) d y_{s},
\end{aligned}
$$

where

$$
\widehat{R}_{E}(\mathbf{w}, k)=\frac{\lambda_{j^{*}}(k)}{\sqrt{2 \pi} \rho_{j^{*}}(k)} \int_{-\infty}^{0} e^{-\frac{\left(y_{s}-\mu_{j^{*}}(k)\right)^{2}}{2 \rho_{j^{*}}^{2}(k)}} d y_{s}=\frac{\lambda_{j^{*}}(k)}{\sqrt{2 \pi}} \int_{g_{j^{*}}(\mathbf{w}, k)}^{\infty} e^{-\frac{y^{2}}{2}} d y
$$

with

$$
g_{j^{*}}(\mathbf{w}, k)=\frac{\mu_{j^{*}}(k)}{\rho_{j^{*}}(k)} .
$$

In (43) only $\mu_{j^{*}}(k)$ and $\rho_{j^{*}}(k)$ depend on the current equalizer's parameter vector $\mathbf{w}$, since only $\mu_{j^{*}}(k)$ and $\rho_{j^{*}}(k)$ depends on $y_{s}(k)=b_{0}(k-d) y(k)=b_{0}(k-d) f(\mathbf{r}(k) ; \mathbf{w})$. Therefore, we have the "instantaneous" gradient of $\widehat{P}_{E}(\mathbf{w}, k)$ given by

$$
\nabla \widehat{P}_{E}(\mathbf{w}, k)=\nabla \widehat{R}_{E}(\mathbf{w}, k)=-\frac{\lambda_{j^{*}}(k)}{\sqrt{2 \pi}} e^{-\frac{\mu_{j^{*}}^{2}(k)}{2 \rho_{j^{*}}^{2}(k)} \frac{\partial g_{j^{*}}(\mathbf{w}, k)}{\partial \mathbf{w}},}
$$

where

$$
\frac{\partial g_{j^{*}}(\mathbf{w}, k)}{\partial \mathbf{w}}=\frac{\left(\rho_{j^{*}}^{2}(k)+\mu_{j^{*}}^{2}(k)\right) \operatorname{sgn}\left(b_{0}(k-d)\right)-\mu_{j^{*}}(k) y(k)}{\rho_{j^{*}}^{3}(k)\left(M \lambda_{j^{*}}(k-1)+1\right)} \frac{\partial f(\mathbf{r}(k) ; \mathbf{w})}{\partial \mathbf{w}},
$$

the derivation of which is given in Appendix B. 
At sample $k$, given the equalizer's signed decision variable $y_{s}(k)=b_{0}(k-d) f(\mathbf{r}(k) ; \mathbf{w}(k-1))$, the on-line parameter updating for the adaptive MBER equalizer based on "stochastic" gradient descent is therefore expressed by

$$
\begin{aligned}
\mathbf{w}(k) & =\mathbf{w}(k-1)-\eta \nabla \widehat{P}_{E}(\mathbf{w}(k-1), k) \\
& =\mathbf{w}(k-1)+\frac{\eta \lambda_{j^{*}}(k)}{\sqrt{2 \pi}} e^{-\frac{\mu_{j^{*}}^{2}(k)}{2 \rho_{j^{*}}^{2}(k)} \frac{\partial g_{j^{*}}(\mathbf{w}(k-1), k)}{\partial \mathbf{w}},}
\end{aligned}
$$

where $\eta>0$ is a small step size.

\section{OMG-MBER Algorithm Summary}

It is well known that the mixture of Gaussians with a small $M$ is capable of accurately estimating an arbitrary PDF [36], [37], [44], [45]. Therefore, the number of mixtures $M$ in the on-line kernel density estimator (28) can be chosen as a very small number, and typically $M=4$ to 6 is sufficient for most applications. The initial means $\mu_{j}(0)$ for $1 \leq j \leq M$ of the PDF estimator can be simply set to some small positive and random values. Our extensive simulation experience suggests that the choice of the initial means $\mu_{j}(0)$ for $1 \leq j \leq M$ are not critical at all to the performance of the adaptive equalizer. This is because the propose OMG PDF estimator has an excellent adaptation capability and is capable of adapting to the underlying true data distribution from different choices of $\mu_{j}(0)$. By the same reason, the choice of the initial kernel width $\rho_{0}$ does not have a major impact on the achievable performance.

The nonlinear equalizer we employed is the RBF equalizer (9). The number of the RBF nodes $n_{c}$ is problem dependent. Specifically, $n_{c}$ is related to the size $N_{r}$ of the state set $\mathbf{R}$. With $n_{c}$ set to $N_{r}$, the RBF equalizer has the potential of attaining the full optimal performance of the Bayesian equalizer (7). However, this is rarely achievable as $N_{r}$ is typically huge. In general, there exists a trade-off between achievable performance and affordable cost in choosing $n_{c}$. A large $n_{c}$ has the potential of achieving a better performance at the cost of higher complexity and slower adaptation process. In practice, we often choose a very small $n_{c}$, compared with the problem size $N_{r}$. We are now ready to summarize the proposed OMG-MBER RBF equalizer.

\section{Initialization $(k \leq 0)$ :}

1) Given the equalizer order $m$ and the RBF equalizer size $n_{c}$, initialize the RBF equalizer, namely, set the initial equalizer's parameter vector $\mathbf{w}(0)$. 
2) Given the number of mixtures $M$ and the initial kernel width $\rho_{0}$, initialize the mixture of Gaussians based PDF estimator (31), namely, set the initial means $\mu_{j}(0), 1 \leq j \leq M$, of the $M$ Gaussians.

Adaptation $(k \geq 1)$ :

1) Given $\mathbf{w}(k-1)$ and the new data $\left\{\mathbf{r}(k), b_{0}(k-d)\right\}$, calculate the RBF equalizer output $y(k)=f(\mathbf{r}(k) ; \mathbf{w}(k-1))$ using (9) and compute the signed decision variable $y_{s}(k)=$ $b_{0}(k-d) y(k)$.

2) Update the mixture of Gaussians based PDF estimator from (32) into (42). Specifically,

- Find the index $j^{*}$ according to (33).

- Update the $j^{*}$-th weight, mean and standard deviation according to (39), (40) and (41), respectively.

- Update the $j$-th weights, means and standard deviations for $1 \leq j \leq M$ and $j \neq j^{*}$ according to (35), (36) and (37), respectively.

3) Given the step size $\eta$, update the RBF equalizer's parameter vector from $\mathbf{w}(k-1)$ to $\mathbf{w}(k)$. Specifically,

- Calculate $\frac{\partial f(\mathbf{r}(k) ; \mathbf{w}(k-1))}{\partial \mathbf{w}}$ according to (10).

- Calculate $\frac{\partial g_{j^{*}}(\mathbf{w}(k-1), k)}{\partial \mathbf{w}}$ according to (47).

- Update the RBF equalizer's parameter vector according to (48).

Because the initial choice of the RBF equalizer's parameter vector w(0) has some influence one the convergence speed of the on-line adaptive procedure, we choose the initial centers $\mathbf{c}_{j}(0)$, $1 \leq j \leq n_{c}$, from the data $\left\{\left(\mathbf{r}(k), b_{0}(k-d)\right), k \leq 0\right\}$ according to the following heuristic rules. Half of the initial centers are chosen from the data points $\mathbf{r}(k)$ that corresponds to the desired signal $b_{0}(k-d)=+1$, while the other half of the initial centers are chosen from the data points $\mathbf{r}(k)$ which has the desired signal value $b_{0}(k-d)=-1$. Moreover, the chosen initial centers must have a minimum distance apart. Specifically, for $1 \leq i, j \leq n_{c}$ and $i \neq j$,

$$
\left\|\mathbf{c}_{j}(0)-\mathbf{c}_{i}(0)\right\|>\frac{\zeta_{0}}{n_{c}}
$$

where the preset positive parameter $\zeta_{0}$ controls the distribution of the initial RBF nodes. The initial weights $\alpha_{j}(0)$ are set either to $+\alpha_{0}$ or to $-\alpha_{0}$, depending on whether $\mathbf{c}_{j}(0)$ are related to $b_{0}(k-d)=+1$ or $b_{0}(k-d)=-1$, where $\alpha_{0}>0$ is a preset parameter. All the initial variances $\sigma_{j}^{2}(0), 1 \leq j \leq n_{c}$, are set to the same value of $\frac{\sigma_{0}^{2}}{n_{c}^{2}}$, where the preset positive value $\sigma_{0}^{2}$ controls 
the influencing field of the initial RBF nodes. This RBF equalizer initialization procedure is similar to the one given in [33].

The values of $\zeta_{0}, \alpha_{0}$ and $\sigma_{0}$ as well as the step size $\eta$ are carefully chosen based on trial-anderror to achieve a best possible performance.

\section{Simulation StUdy}

Two examples were used to compare the proposed OMG-MBER RBF equalizer with the LBER RBF equalizer given in [33] and the conventional linear MMSE equalizer based on the LMS algorithm. For the both examples, the number of mixtures was set to $M=6$ with the initial kernel parameters $\rho_{0}=1$ and $\mu_{j}(0), 1 \leq j \leq M$, randomly chosen from [0.5, 1.5] for the proposed OMG PDF estimator, while the step size of the OMG-MBER RBF equalizer was set to $\eta=0.3$.

Example One. In the first experiment, the transfer functions of the channel and co-channel were given as $A_{0}(z)=0.5+1.0 z^{-1}$ and $A_{1}(z)=\lambda\left(1.0+0.5 z^{-1}\right)$, respectively, where $\lambda$ was set to yield the signal to interference ratio (SIR) of $12 \mathrm{~dB}$. The equalizer order and decision delay were set to $m=2$ and $d=1$, respectively, and therefore the size of the state set $\mathbf{R}$ was $N_{r}=64$. For both the OMG-MBER and LBER based RBF equalizers, we had $n_{c}=4$. For the OMG-MBER RBF equalizer, the initialization was carried out with $\zeta_{0}=8, \sigma_{0}=6$ and $\alpha_{0}=10$. For the LBER RBF equalizer, the same initialization as in [33] was used and moreover the LBER algorithmic parameters were set to $\eta_{k}=0.5 k^{-0.25}$ and $\rho^{2}=20 \sigma_{n}^{2}$, the same as in [33].

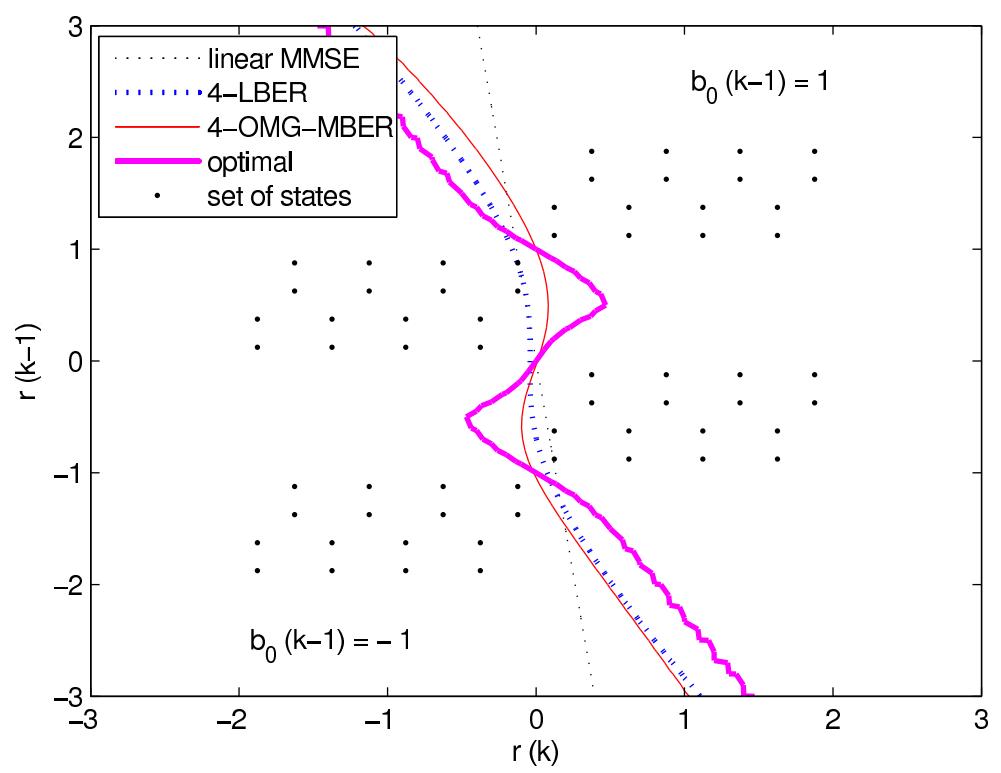

Fig. 1. Comparison of decision boundaries (thin dotted: linear MMSE, thick dotted: LBER RBF, thin solid: proposed OMGMBER RBF, and thick solid: optimal Bayesian) for Example 1 given $\mathrm{SNR}=15 \mathrm{~dB}$. 


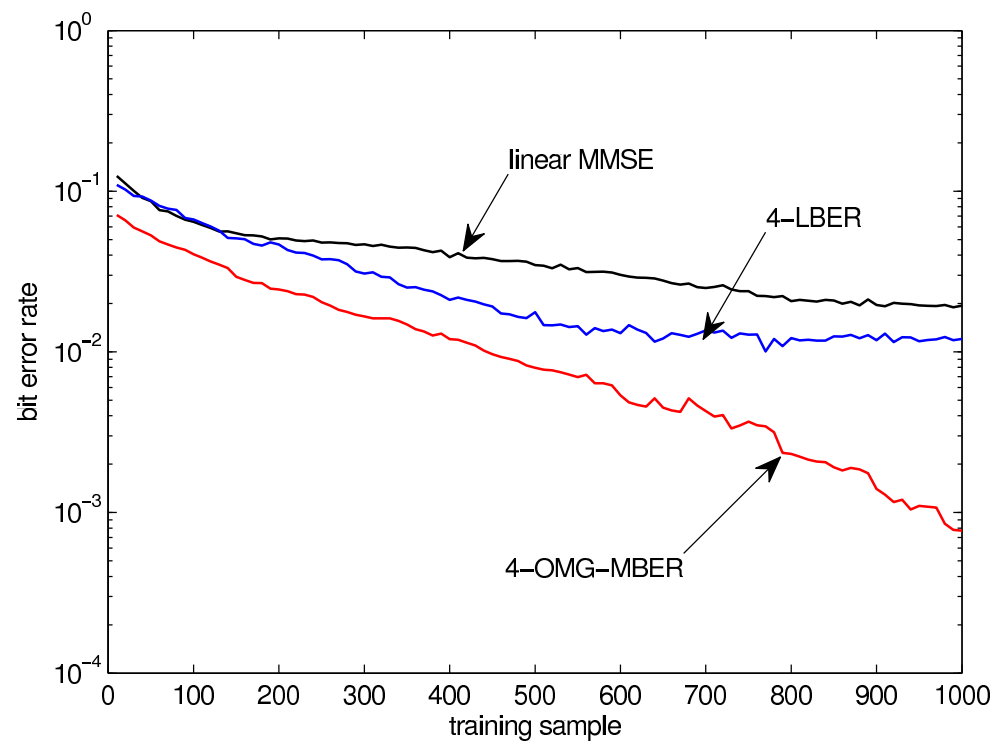

Fig. 2. Comparison of learning curves for the linear MMSE equalizer based on the LMS, the LBER RBF equalizer and the OMG-MBER RBF equalizer, in terms of BER performance and averaged over 100 runs for Example 1 given SNR=15 dB.

Fig. 1 compares the decision boundaries of the linear MMSE equalizer, the LBER RBF equalizer and the proposed OMG-MBER RBF equalizer using the optimal Bayesian equalizer (7) as the benchmark, under the signal to noise ratio (SNR) condition of $15 \mathrm{~dB}$. It can be clearly seen from Fig. 1 that the linear MMSE equalizer could only realize a linear decision boundary, while the LBER and OMG-MBER RBF equalizers with only 4 RBF nodes were capable of approximating well the optimal nonlinear decision boundary of the 64-nodes Bayesian equalizer. The learning curves of the four-center LBER and OMG-MBER RBF equalizers as well as the

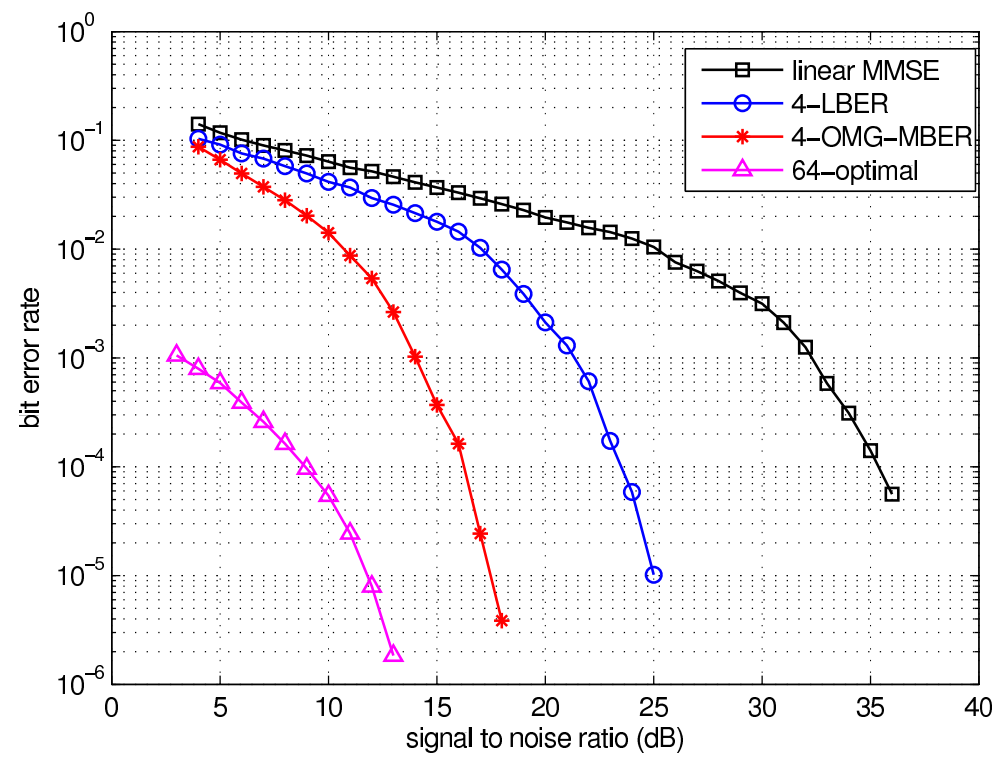

Fig. 3. BER performance comparison of the linear MMSE equalizer based on the LMS, the LBER RBF equalizer and the OMG-MBER RBF equalizer as well as the optimal Bayesian equalizer for Example 1. 
two-tap linear MMSE equalizer based on the LMS algorithm are compared in Fig. 2 given $\mathrm{SNR}=15 \mathrm{~dB}$, where the BER results were averaged over 100 runs. As expected, the linear MMSE equalizer had the worst BER performance. From Fig. 2, it can be seen that the OMGMBER RBF equalizer considerably outperformed the LBER RBF equalizer both in convergence speed and achievable BER performance. The achievable BER performance of the three adaptive equalizers are depicted in Fig. 3 together with the BER of the optimal Bayesian equalizer. The results of Fig. 3 again confirm that the proposed OMG-MBER RBF equalizer had the superior performance over the LBER RBF equalizer.

Example Two. The transfer functions of the channel and co-channel were specified by $A_{0}(z)=$ $0.3482+0.8704 z^{-1}+0.3482 z^{-2}$ and $A_{1}(z)=\lambda\left(0.6+0.8 z^{-1}\right)$, respectively, where the value of $\lambda$ was chosen to yield the $\mathrm{SIR}=20 \mathrm{~dB}$. The equalizer order was set to $m=4$ and the decision delay $d=1$. The number of the signal states in $\mathbf{R}$ was $N_{r}=2048$. We used $n_{c}=16$ for both the LBER and OMG-MBER RBF equalizers. For the initialization of the OMG-MBER RBF equalizer, we used $\zeta_{0}=12, \sigma_{0}=16$ and $\alpha_{0}=10$. For the LBER RBF equalizer, $\eta_{k}=0.3 k^{-0.25}$ and $\rho^{2}=10 \sigma_{n}^{2}$ as well as the same initialization as given in [33] were used.

The learning curves of the three adaptive equalizers are shown in Fig. 4 given the $\mathrm{SNR}=$ $15 \mathrm{~dB}$, where the results were averaged over 100 runs. As expected, the 4-tap linear MMSE equalizer trained by the LMS algorithm had the fastest convergence speed but its achievable BER performance was the worst. Fig. 5 depicts the BER performance of the three adaptive equalizers tested. From both Figs. 4 and 5, it can be clearly seen that the proposed OMG-

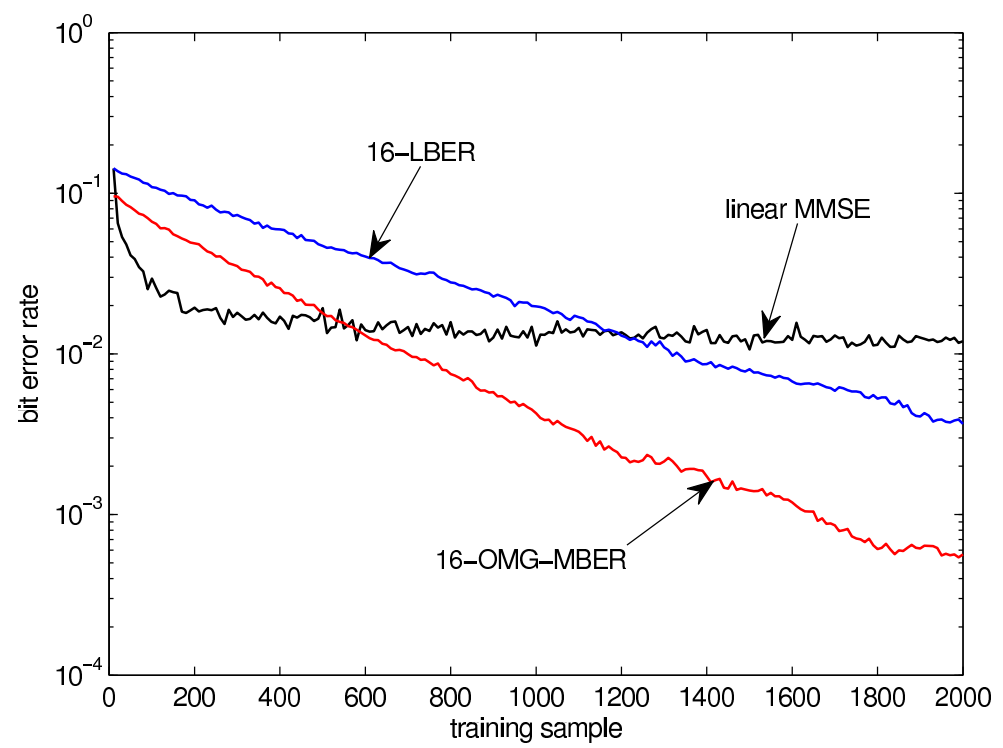

Fig. 4. Comparison of learning curves for the linear MMSE equalizer based on the LMS, the LBER RBF equalizer and the OMG-MBER RBF equalizer, in terms of BER performance and averaged over 100 runs for Example 2 given $\mathrm{SNR}=15 \mathrm{~dB}$. 


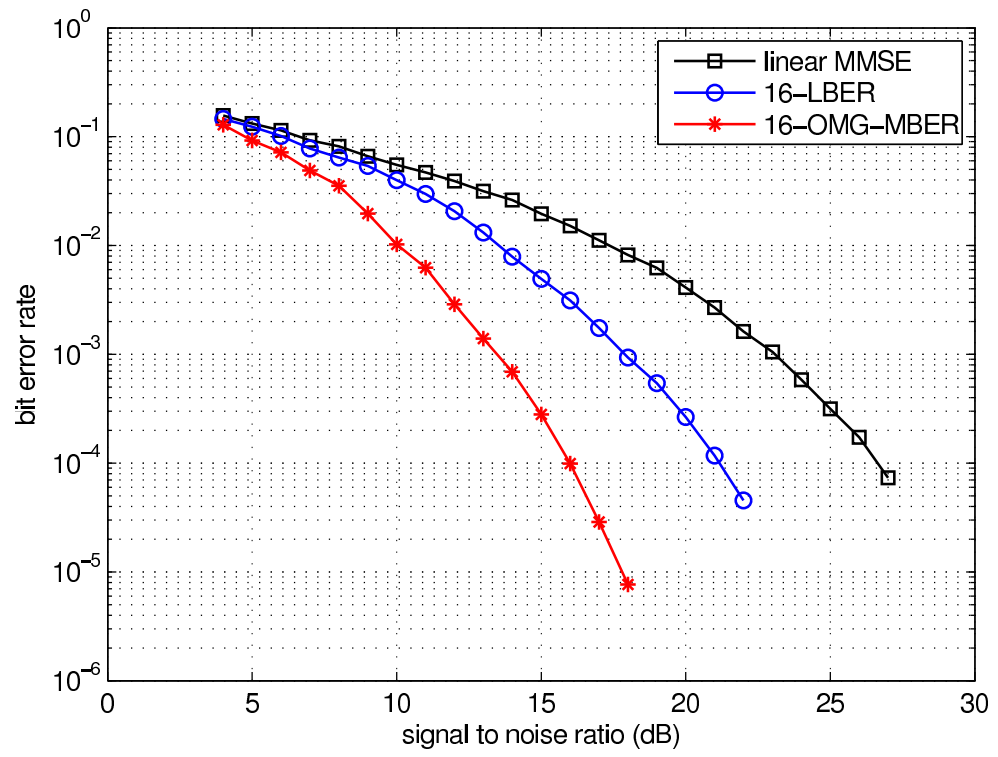

Fig. 5. BER performance comparison of the linear MMSE equalizer based on the LMS, the LBER RBF equalizer and the OMG-MBER RBF equalizer for Example 2.

MBER RBF equalizer significantly outperformed the LBER RBF equalizer, in terms of both convergence speed and achievable BER. It is worth noting that for this example the optimal full Bayesian solution would require 2048 nodes. Thus, this optimal Bayesian equalizer becomes impractical when the channel and interfering co-channel both have high orders.

\section{CONCLUSions}

The performance of an adaptive MBER nonlinear equalizer critically depends on the on-line PDF estimator used for estimating the signed decision variable's distribution. In this contribution, a new on-line small-size Gaussian mixture based PDF estimator has been proposed to aid an adaptive MBER nonlinear equalizer. The resulting on-line MBER nonlinear equalizer, referred to as the OMG-MBER nonlinear equalizer, has been introduced under the generic setting of intersymbol interference channel plus co-channel interference. Compared with the existing LBER nonlinear equalizer, which relies on a one-sample PDF estimator, our on-line mixture of Gaussians based PDF estimator is capable of providing a much more accurate estimate of the signed decision variable's PDF while maintaining a very low computational requirements for online PDF estimation. Consequently, our proposed OMG-MBER nonlinear equalizer significantly outperforms the LBER nonlinear equalizer, in terms of both convergence speed and achievable BER performance. 


\section{APPENDIX}

\section{A. Merging Two Gaussians as One}

Consider merging a mixture of two Gaussians

$$
\widehat{p}^{(2)}\left(y_{s} ; \boldsymbol{\lambda}_{2}, \boldsymbol{\mu}_{2}, \boldsymbol{\rho}_{2}\right)=\sum_{j=1}^{2} \lambda_{j} G\left(y_{s} ; \mu_{j}, \rho_{j}\right)
$$

into one mixture by matching the resultant mean and variance. The mean $\mu$ of the two mixtures is given by

$$
\mu=\int y_{s} \widehat{p}^{(2)}\left(y_{s} ; \boldsymbol{\lambda}_{2}, \boldsymbol{\mu}_{2}, \boldsymbol{\rho}_{2}\right) d y_{s}=\sum_{j=1}^{2} \lambda_{j} \int y_{s} G\left(y_{s} ; \mu_{j}, \rho_{j}\right) d y_{s}=\sum_{j=1}^{2} \lambda_{j} \mu_{j},
$$

while the variance $\rho^{2}$ of the two mixtures is

$$
\begin{aligned}
\rho^{2} & =\int\left(y_{s}-\mu\right)^{2} \widehat{p}^{(2)}\left(y_{s} ; \boldsymbol{\lambda}_{2}, \boldsymbol{\mu}_{2}, \boldsymbol{\rho}_{2}\right) d y_{s}=\int y_{s}^{2} \widehat{p}^{(2)}\left(y_{s} ; \boldsymbol{\lambda}_{2}, \boldsymbol{\mu}_{2}, \boldsymbol{\rho}_{2}\right) d y_{s}-\mu^{2} \\
& =\sum_{j=1}^{2} \lambda_{j} \int y_{s}^{2} G\left(y_{s}, \mu_{j}, \rho_{j}\right) d y_{s}-\mu^{2}=\sum_{j=1}^{2} \lambda_{j}\left(\rho_{j}^{2}+\mu_{j}^{2}\right)-\mu^{2} .
\end{aligned}
$$

B. The Derivation of $\frac{\partial g_{j^{*}}(\mathbf{w}, k)}{\partial \mathbf{w}}$

Noting $y_{s}(k)=\operatorname{sgn}\left(b_{0}(k-d)\right) f(\mathbf{r}(k) ; \mathbf{w})$, (40) becomes

$$
\mu_{j^{*}}(k)=\frac{M \lambda_{j^{*}}(k-1)}{M \lambda_{j^{*}}(k-1)+1} \mu_{j^{*}}(k-1)+\frac{\operatorname{sgn}\left(b_{0}(k-d)\right)}{M \lambda_{j^{*}}(k-1)+1} f(\mathbf{r}(k) ; \mathbf{w}) .
$$

The partial derivative of $\mu_{j^{*}}(k)$ with respect to the equalizer's parameter vector $\mathbf{w}$, denoted as $\mu_{j^{*}}^{\prime}(k)$, is then given by

$$
\mu_{j^{*}}^{\prime}(k)=\frac{\partial \mu_{j^{*}}(k)}{\partial \mathbf{w}}=\frac{\operatorname{sgn}\left(b_{0}(k-d)\right)}{M \lambda_{j^{*}}(k-1)+1} \frac{\partial f(\mathbf{r}(k) ; \mathbf{w})}{\partial \mathbf{w}} .
$$

From (41), we also have

$$
\begin{aligned}
\rho_{j^{*}}^{2}(k) & =\frac{M \lambda_{j^{*}}(k-1)\left(\rho_{j^{*}}^{2}(k-1)+\mu_{j^{*}}^{2}(k-1)\right)}{M \lambda_{j^{*}}(k-1)+1}+\frac{\rho_{0}^{2}+\left(\operatorname{sgn}\left(b_{0}(k-d)\right) f(\mathbf{r}(k) ; \mathbf{w})\right)^{2}}{M \lambda_{j^{*}}(k-1)+1}-\mu_{j^{*}}^{2}(k) \\
& =\frac{M \lambda_{j^{*}}(k-1)\left(\rho_{j^{*}}^{2}(k-1)+\mu_{j^{*}}^{2}(k-1)\right)}{M \lambda_{j^{*}}(k-1)+1}+\frac{\rho_{0}^{2}+f^{2}(\mathbf{r}(k) ; \mathbf{w})}{M \lambda_{j^{*}}(k-1)+1}-\mu_{j^{*}}^{2}(k)
\end{aligned}
$$

Thus the partial derivation of $\rho_{j^{*}}^{2}(k)$ with respect to the equalizer's parameter vector $\mathbf{w}$ is

$$
\frac{\partial \rho_{j^{*}}^{2}(k)}{\partial \mathbf{w}}=\frac{2 y(k)}{M \lambda_{j^{*}}(k-1)+1} \frac{\partial f(\mathbf{r}(k) ; \mathbf{w})}{\partial \mathbf{w}}-2 \mu_{j^{*}}(k) \frac{\partial \mu_{j^{*}}(k)}{\partial \mathbf{w}} .
$$


Substituting (54) into (56) yields

$$
\begin{aligned}
\frac{\partial \rho_{j^{*}}^{2}(k)}{\partial \mathbf{w}} & =\frac{2 y(k)}{M \lambda_{j^{*}}(k-1)+1} \frac{\partial f(\mathbf{r}(k) ; \mathbf{w})}{\partial \mathbf{w}}-\frac{2 \mu_{j^{*}}(k) \operatorname{sgn}\left(b_{0}(k-d)\right)}{M \lambda_{j^{*}}(k-1)+1} \frac{\partial f(\mathbf{r}(k) ; \mathbf{w})}{\partial \mathbf{w}} \\
& =\frac{2\left(y(k)-\mu_{j^{*}}(k) \operatorname{sgn}\left(b_{0}(k-d)\right)\right)}{M \lambda_{j^{*}}(k-1)+1} \frac{\partial f(\mathbf{r}(k) ; \mathbf{w})}{\partial \mathbf{w}} .
\end{aligned}
$$

Denoting $\rho_{j^{*}}^{\prime}(k)=\frac{\partial \rho_{j^{*}}(k)}{\partial \mathbf{w}}$, then

$$
\frac{\partial \rho_{j^{*}}^{2}(k)}{\partial \mathbf{w}}=2 \rho_{j^{*}}(k) \rho_{j^{*}}^{\prime}(k)
$$

From (57) and (58), we have

$$
\rho_{j^{*}}^{\prime}(k)=\frac{y(k)-\mu_{j^{*}}(k) \operatorname{sgn}\left(b_{0}(k-d)\right)}{\rho_{j^{*}}(k)\left(M \lambda_{j^{*}}(k-1)+1\right)} \frac{\partial f(\mathbf{r}(k) ; \mathbf{w})}{\partial \mathbf{w}} .
$$

Finally by making use of (59) and (54), we have

$$
\begin{aligned}
\frac{\partial g_{j^{*}}(\mathbf{w}, k)}{\partial \mathbf{w}} & =\frac{\mu_{j^{*}}^{\prime}(k) \rho_{j^{*}}(k)-\mu_{j^{*}}(k) \rho_{j^{*}}^{\prime}(k)}{\rho_{j^{*}}^{2}(k)} \\
& =\frac{\left(\rho_{j^{*}}^{2}(k)+\mu_{j^{*}}^{2}(k)\right) \operatorname{sgn}\left(b_{0}(k-d)\right)-\mu_{j^{*}}(k) y(k)}{\rho_{j^{*}}^{3}(k)\left(M \lambda_{j^{*}}(k-1)+1\right)} \frac{\partial f(\mathbf{r}(k) ; \mathbf{w})}{\partial \mathbf{w}} .
\end{aligned}
$$

\section{REFERENCES}

[1] S. Qureshi, “Adaptive equalization,” Proceedings of the IEEE, vol. 73, no. 9, pp. 1349-1387, Sept. 1985.

[2] G. D. Forney Jr, "Maximum-likelihood sequence estimation of digital sequences in the presence of intersymbol interference," IEEE Transactions on Information Theory, vol. 18, no. 3, pp. 363-378, May 1972.

[3] D. Williamson, R. A. Kennedy, and G. W. Pulford, "Block decision feedback equalization," IEEE Transactions on Communications, vol. 40, no. 2, pp. 255-264, Feb. 1992.

[4] S. Chen, B. Mulgrew, and S. McLaughlin, "Adaptive Bayesian equalizer with decision feedback," IEEE Transactions on Signal Processing, vol. 41, no. 9, pp. 2918-2927, Sept. 1993.

[5] S. Siu, G. J. Gibson, and C. F. N. Cowan, "Decision feedback equalisation using neural network structures and performance comparison with standard architecture," IEE Proceedings I - Communications, Speech and Vision, vol. 137, no. 4, pp. 221225, Aug. 1990.

[6] S. Chen, G. J. Gibson, and C. F. N. Cowan, “Adaptive channel equalisation using a polynomial-perceptron structure,” IEE Proceedings I - Communications, Speech and Vision, vol. 137, no. 5, pp. 257-264, Oct. 1990.

[7] G. J. Gibson, S. Siu, and C. F. N. Cowan, "The application of nonlinear structures to the reconstruction of binary signals," IEEE Transactions on Signal Processing, vol. 39, no. 8, pp. 1877-1884, Aug. 1991.

[8] S. Chen, G. J. Gibson, C. F. N. Cowan, and P. M. Grant, "Reconstruction of binary signals using an adaptive radial-basisfunction equalizer," Signal Processing, vol. 22, no. 1, pp. 77-93, Jan. 1991.

[9] I. Cha and S. Kassam, "Channel equalization using adaptive complex radial basis function networks," IEEE Journal on Selected Areas in Communications, vol. 13, no. 1, pp. 122-131, Jan. 1995.

[10] S. Haykin, Adaptive Filter Theory (2nd Edition). Englewood, NJ: Prentice Hall, 1991. 
[11] B. Widrow and M. E. Hoff, "Adaptive switching circuits," in 1960 IRE WESCON Convention Record, 1960, Part 4, pp. 96-104.

[12] E. Shamash and K. Yao, "On the structure and performance of a linear decision feedback equalizer based on the minimum error probability criterion," in Proceedings of 1974 IEEE International Conference on Communications (Minneapolis, USA), June 17-19, 1974, pp. 25F1-25F5.

[13] S. Chen, E. S. Chng, B. Mulgrew, and G. J. Gibson, "Minimum-BER linear-combiner DFE," in Proceedings of 1996 IEEE International Conference on Communications, (Dallas, TX), June 23-27, 1996, vol. 2, pp. 1173-1177.

[14] C.-C. Yeh, R. R. Lopes, and J. R. Barry, "Approximate minimum bit-error rate multiuser detection," in Proceedings of 1998 IEEE Global Telecommunications Conference (Sydney, NSW ), Nov. 8-12, 1998, vol. 6, pp. 3590-3595.

[15] S. Chen, B. Mulgrew, E. S. Chng, and G. J. Gibson, "Space translation properties and the minimum-BER linear-combiner DFE,” IEE Proceedings - Communications, vol. 145, no. 5, pp. 316-322, Oct. 1998.

[16] S. Chen and B. Mulgrew, "Minimum-SER linear-combiner decision feedback equaliser," IEE Proceedings - Communications, vol. 146, no. 6, pp. 347-353, Dec. 1999.

[17] I. N. Psaromiligkos, S. N. Batalama and D. A. Pados, "On adaptive minimum probability of error linear filter receivers for DS-CDMA channels," IEEE Transactions on Communications, vol. 47, no. 7, pp. 1092-1102, July 1999.

[18] S. Chen, S. Gunn, and C. J. Harris, "Decision feedback equaliser design using support vector machines," IEE Proceedings - Vision, Image and Signal Processing, vol. 147, no. 3, pp. 213-219, June 2000.

[19] X. F. Wang, W.-S. Lu, and A. Antoniou, “Constrained minimum-BER multiuser detection,” IEEE Transactions on Signal Processing, vol. 48, no. 10, pp. 2903-2909, Oct. 2000.

[20] C.-C. Yeh and J. R. Barry, "Adaptive minimum bit-error rate equalization for binary signaling," IEEE Transactions on Communications, vol. 48, no. 7, pp. 1226-1235, July 2000.

[21] B. Mulgrew and S. Chen, "Stochastic gradient minimum-BER decision feedback equalisers," in Proceedings of 2000 IEEE Adaptive Systems for Signal Processing, Communications, and Control Symposium (Lake Louise, Canada), Oct. 1-4, 2000, pp. 93-98.

[22] K. A. Phillips, J. H. Reed, and W. H. Tranter, "Minimum BER adaptive filtering," in Proceedings of 2000 IEEE International Conference on Communications (New Orleans, LA), June 18-22, 2000, Vol. 3, pp. 1675-1680.

[23] B. Mulgrew and S. Chen, "Adaptive minimum-BER decision feedback equalisers for binary signalling," Signal Processing, vol. 81, no. 7, pp. 1479-1489, July 2001.

[24] S. Chen, L. Hanzo, and N. N. Ahmad, "Adaptive minimum bit error rate beamforming assisted receiver for wireless communications," in Proceedings of 2003 IEEE International Conference on Acoustics, Speech, and Signal Processing (Hong Kong, China), April 6-10, 2003, vol. IV, pp. 640-643.

[25] M. Y. Alias, A. K. Samingan, S. Chen, and L. Hanzo, "Multiple antenna aided OFDM employing minimum bit error rate multiuser detection," Electronics Letters, vol. 39, no. 24, pp. 1769-1770, 2003.

[26] S. J. Yi, C. C. Tsimenidis, O. R. Hinton, and B. S. Sharif, "Adaptive minimum bit error rate multiuser detection for asynchronous MC-CDMA systems frequency selective Rayleigh fading channels," in Proceedings of 14th IEEE International Symposium on Personal, Indoor and Mobile Radio Communications (Beijing, China ), Sept.7-10, 2003, vol. 2, pp. 1269-1273.

[27] R. C. de Lamare and R. Sampaio-Neto, "Adaptive MBER decision feedback multiuser receivers in frequency selective fading channels," IEEE Communications Letters, vol. 7, no. 2, pp. 73-75, Feb. 2003.

[28] D. Gesber, "Robust linear MIMO receivers: a minimum error-rate approach," IEEE Transactions on Signal Processing, vol. 51, no. 11, pp. 2863-2871, Nov. 2003.

[29] F.-J. Chen, S.-K. Xiong, and G. Wei, "A new constrained minimum-BER multiuser detection algorithm," in Proceedings of 
IEEE 6th Circuits and Systems Symposium on Emerging Technologies: Frontiers of Mobile and Wireless Communication (Shanghai, China), 1 May-2 June, 2004, vol. 3, pp. 365-368.1.

[30] S. Chen, N. N. Ahmad, and L. Hanzo, "Adaptive minimum bit error rate beamforming," IEEE Transactions on Wireless Communications, vol. 4, no. 2, pp. 341-348, March 2005.

[31] S. Chen, A. Livingstone, and L. Hanzo, "Minimum bite-error rate design for space-time equalization-based multiuser detection," IEEE Transaction on Communications, vol. 54, no. 5, pp. 824-832, May 2006.

[32] J. Riani, S. van Beneden, J. W. M. Bergmans, and A. Immink, "Near-minimum bit-error rate equalizer adaptation for PRML systems," IEEE Transactions on Communications, vol. 55, no. 12, pp. 2316-2327, Dec. 2007

[33] S. Chen, B. Mulgrew, and L. Hanzo, "Least bit error rate adaptive nonlinear equalisers for binary signalling," IEE Proceedings - Communications, vol. 150, no. 1, pp. 29-36, Feb. 2003.

[34] S. Chen, "Adaptive minimum bit-error-rate filtering," IEE Proceedings - Vision, Image and Signal Processing, vol. 151, no. 1, pp. 76-85, Feb. 2004.

[35] L. Kovacs, J. Levendovszky, A. Olah, and G. Treplan, "Approximate minimum bit error rate equalization for fading channels," EURASIP Journal on Advances in Signal Processing, vol. 2010, Article No. 7, 9 pages, Feb. 2010.

[36] J. A. Bilmes, "A gentle tutorial of the EM algorithm and its application to parameter estimation for Gaussian mixture and hidden Markov models," International Computer Science Institute, vol. 4, no. 510, 281 pages, 1998.

[37] G. McLachlan and D. Peel, Finite Mixture Models. John Wiley \& Sons, Inc., 2004.

[38] S. Chen, X. Hong, and C. J. Harris, "Sparse kernel density construction using orthogonal forward regression with leaveone-out test score and local regularization," IEEE Transactions on Systems, Man, and Cybernetics, Part B: Cybernetics, vol. 34, no. 4, pp. 1708-1717, Aug. 2004.

[39] X. Hong, S. Chen, and C. J. Harris, "A forward-constrained regression algorithm for sparse kernel density estimation," IEEE Transactions on Neural Networks, vol. 19, no. 1, pp. 193-198, Jan. 2008.

[40] S. Chen, X. Hong, and C. J. Harris, "An orthogonal forward regression technique for sparse kernel density estimation," Neurocomputing, vol. 71, nos. 4-6, pp. 931-943, Jan. 2008.

[41] S. Chen, X. Hong, and C. J. Harris, "Regression based D-optimality experimental design for sparse kernel density estimation," Neurocomputing, vol. 73, nos. 4-6, pp. 727-739, Jan. 2010.

[42] S. Chen, S. McLaughlin, B. Mulgrew, and P. M. Grant, "Bayesian decision feedback equaliser for overcoming co-channel interference," IEE Proceedings - Communications, vol. 143, no. 4, pp. 219-225, Aug. 1996.

[43] S. Chen, B. Mulgrew, and P. M. Grant, "A clustering technique for digital communications channel equalization using radial basis function networks," IEEE Transactions on Neural Networks, vol. 4, no. 4, pp. 570-590, July 1993.

[44] S. Chen, X. Hong, B. L. Luk, and C. J. Harris, "Construction of tunable radial basis function networks using orthogonal forward selection," IEEE Transactions on Systems, Man, and Cybernetics, Part B: Cybernetics, vol. 39, no. 2, pp. 457-466, April 2009.

[45] S. Chen, X. Hong and C. J. Harris, "Particle swarm optimization aided orthogonal forward regression for unified data modelling," IEEE Transactions on Evolutionary Computation, vol. 14, no. 4, pp. 477-499, Aug. 2010. 